\title{
PERFIL EPIDEMIOLÓGICO DE PACIENTES ATENDIDOS EM UMA REDE AMBULATORIAL DO HIPERDIA MINAS EM GOVERNADOR VALADARES-MG
}

\author{
EPIDEMIOLOGICAL PROFILE OF PATIENTS WHO RECEIVED OUTPATIENT CAREAT HIPERDIA \\ MINAS CENTER IN GOVERNADOR VALADARES, MINAS GERAIS
}

\section{Ludmilla Grossi Furtado Saraivaa , Patrícia Grossi Dornelas ${ }^{b}$, Stefany Bruno de Assis Cauc, Luciana Karen Calábriad}

aludmillasaraiva@yahoo.com.br, bpatriciagrossid@hotmail.com, ccau@icb.ufmg.br, ${ }^{\mathrm{d}}$ lkcalabria@ufu.br Universidade Federal de Juiz de Fora - Campus de Governador Valadares (MG), Brasil

Data de recebimento do artigo: 29/08/2015 Data de aceite do artigo: 19/02/2016

\section{RESUMO}

Introduçãa: As doenças crônicas não transmissíveis, como a hipertensão arterial e o diabetes mellitus, têm sido causa do aumento no número de óbitos e da perda da qualidade de vida. Objetivo: Considerando a importância das doenças crônicas não transmissíveis e das açôes do Programa Hiperdia, realizou-se um estudo epidemiológico da população diabética e/ou hipertensa. Materiais e métodos: Estudo transversal realizado em Governador Valadares-MG, com 315 adultos ( $\geq 18$ anos) entrevistados na Policlínica Central da cidade utilizando questionário semiestruturado. Resultados: $\mathrm{O}$ diagnóstico sociodemográfico revelou maioria do sexo feminino, idosa, aposentada, com baixa renda e baixo nível de escolaridade. Do total, $47,6 \%$ são diabéticos, $83,8 \%$ hipertensos e $31,4 \%$ com ambas as condiçóes, sendo as mulheres maioria nessas variáveis. Enquanto $100 \%$ dos hipertensos e $83,3 \%$ dos diabéticos revelaram usar medicamentos, com destaque ao losartano e a metformina, apenas $41,9 \%$ praticavam atividade física, sendo a caminhada a modalidade mais frequentemente citada. Além disso, vale ressaltar que $31,4 \%$ dos entrevistados relataram fazer uso da associação de fármacos, anti-hipertensivo e antidiabéticos. Conclusão: A população usuária do Programa Hiperdia Minas em Governador Valadares-MG é suscetível, idosa, polimedicada, e de baixo grau de instrução e poder aquisitivo. Uma vez que a intervençáo terapêutica da equipe de saúde se baseia no tratamento medicamentoso, incentivos na mudança do estilo de vida se fazem necessários para diminuição de fatores de risco nessas doenças crônicas.

Palavras-chave: Doenças crônicas; fatores de risco; polimedicação.

\section{ABSTRACT}

Introduction: Chronic non-communicable diseases, such as hypertension and diabetes mellitus, have been responsible for increasing number of deaths and for loss of quality of life. Objective: Bearing in mind the importance of chronic non-communicable diseases and of actions promoted by Hiperdia Program, an epidemiological study was carried out on diabetic and/or hypertensive population. Material and methods: Cross-sectional study conducted in Governador Valadares, Minas Gerais, with 315 adults (aged $\geq 18$ years) interviewed on Central Polyclinic using semi-structured questionnaire. Results: 'The sociodemographic diagnosis revealed a majority of elderly, retired, with low income and low educational level women. $47.6 \%$ of the total respondents are diabetics, $83.8 \%$ had hypertension, $31.4 \%$ had both conditions, most of them women. While $100 \%$ of high blood pressure patients and $83.3 \%$ of patients with diabetes revealed that they were taking medication, especially losartan and metformin, only $41.9 \%$ practiced physical activity and walking was most frequently cited. In addition, $31.4 \%$ of total respondents have taken combined medication, antihypertensive and antidiabetic. Conclusion: the user population of Hiperdia Minas Program in Governador Valadares, Minas Gerais, is susceptible, elderly, polymedicated, and with low level of education and income. Once the therapeutic intervention of the health team is based on drug treatment, incentives for lifestyle change are necessary to reduce chronic diseases risk factors.

Keywords: Chronic diseases; risk factors; polymedication. 


\section{Introdução}

As mudanças ocorridas no perfil demográfico brasileiro, como o aumento substancial do número de pessoas idosas, são acompanhadas de modificações no cenário nutricional e epidemiológico dessa população, predominando as doenças crônicas não transmissíveis, como a hipertensão arterial sistêmica e o diabetes mellitus, próprias das faixas etárias mais avançadas ${ }^{1}$.

A hipertensão arterial sistêmica (HAS) é um grave problema de saúde pública. Em 2011, cerca de 7,6 milhóes de mortes no mundo foram atribuídas à elevação da pressão arterial, sendo $54 \%$ por acidente vascular encefálico e $46 \%$ por doença isquêmica do coração. No Brasil, nos últimos 20 anos estima-se uma prevalência de HAS acima de $30 \%$, sendo que na faixa etária de 60 a 69 anos corresponde a mais de $50 \%$, e acima de 70 anos, mais de $75 \%{ }^{2}$.

Já o diabetes mellitus (DM) destaca-se entre as condiçôes crônicas mais relevantes de saúde devido à alta prevalência e morbimortalidade ${ }^{3}$. No período de 2012, segundo informaçóes do DATASUS, houve uma prevalência de $11,7 \%$ de adultos diabéticos com 35 anos ou mais, e $23 \%$ na faixa etária acima de 65 anos $^{4}$. O DM aparece como a sexta causa de internação hospitalar, correspondendo a $30 \%$ dos pacientes internados em unidades coronarianas intensivas e $26 \%$ dos que ingressam em programas de hemodiálise ${ }^{3}$.

Assim, a HAS e o DM representam uma das principais causas de óbito no país, e acomete a população mundial em todas as faixas etárias, cujo número de casos atualmente adquire proporção de pandemia. Dentre os fatores que contribuem para o agravo desse quadro epidemiológico destacam-se a crescente longevidade da populaçáo; os maus hábitos de vida como sedentarismo, tabagismo e alimentação inadequada; além do despreparo e da falta de recursos do governo para deter a progressão dessas doenças 5 . Medidas preventivas e curativas relativamente simples, como mudanças no estilo de vida do indivíduo, são necessárias para o controle de ambas as condiçôes clínicas. No que se refere ao trabalho de prevenção, detecção e tratamento em estância pública, faz-se essencial a aplicação de estudos epidemiológicos, propiciando o desenvolvimento de campanhas educativas, o diagnóstico precoce e o uso racional de recursos financeiros para o tratamento dessas doenças 5 .

Nesse sentido, no Brasil, com o objetivo de reduzir a morbimortalidade associada à HAS e ao DM, o Ministério da Saúde assumiu parcerias com estados, municípios e sociedade, dando suporte para a melhoria na atenção aos portadores dessas doenças ${ }^{5}$. Dentro dessa perspectiva, encontra-se o Programa Hiperdia, que permitiu cadastrar e acompanhar os indivíduos diabéticos e/ou hipertensos captados e vinculados às Unidades
Básicas de Saúde (UBS) ou Equipes de Estratégia da Saúde da Família (ESF) do Sistema Único de Saúde (SUS), sendo possível definir um perfil epidemiológico da população $o^{1,6}$ e, consequentemente, desenvolver políticas públicas de saúde que levem à melhoria da qualidade de vida dessas pessoas, tendo como base a análise dos seus dados. Além disso, o Hiperdia teve como objetivo informar os pacientes para a aquisição, dispensação e distribuição de medicamentos de forma sistemática.

No estado de Minas Gerais, estima-se que existam cerca de 540 mil diabéticos, dentre eles 670 mil com $\mathrm{HAS}^{7}$. Segundo esses dados, o município de Governador Valadares apresenta $6,5 \%$ dos diabéticos cadastrados no SisHiperdia (cerca de 1.500 pacientes), sendo 29,2\% cadastrados como diabéticos com HAS (cerca de 6.400 pacientes).

O município de Governador Valadares está localizado na regiâo leste do estado de Minas Gerais, conta com 263.689 habitantes em 2010 e 275.568 estimados para $2013^{8}$, apresenta $11,7 \%$ da sua população na faixa etária de 60 anos e mais. Comparando o Censo dos últimos 10 anos percebe-se que há um envelhecimento populacional crescente no município com uma média de 850 novos idosos a cada ano. As doenças do aparelho circulatório foram associadas a $29,8 \%$ das internaçóes e corresponderam a $40,2 \%$ das causas de mortalidade para esta faixa etária. Em relação às principais internaçôes realizadas no ano e a mortalidade registrada para essa faixa etária, constata-se que a principal causa de internação está associada às doenças do aparelho circulatório com prevalência de $29,8 \%$ e $40,2 \%$, respectivamente. Além disso, houve um aumento progressivo do coeficiente de mortalidade para o infarto agudo do miocárdio $(29,1 \%)$, doenças cerebrovasculares $(12,4 \%)$ e DM $(20,6 \%)$ como causas de óbito no período de 2002 a $2008^{4}$.

Considerando a importância epidemiológica das doenças crônicas não transmissíveis, como o DM e a HAS, bem como das açóes do Programa Hiperdia, o objetivo central deste estudo foi levantar as características sociodemográficas, os medicamentos prescritos e o estilo de vida da população diabética e/ou hipertensa que frequenta uma das redes ambulatoriais do Hiperdia Minas em Governador Valadares, visando a caracterizar os usuários do programa e, assim, conhecer o perfil e as necessidades dos usuários nesse município.

\section{Métodos}

Com delineamento transversal e utilizando dados primários, a população-alvo foi constituída por indivíduos com idade superior a 18 anos, diabéticos e/ou hipertensos, atendidos pela Policlínica Central Municipal 
Josephina Perim de Tassis, Governador Valadares. Os dados foram coletados por meio de entrevista semiestruturada direta, sendo todas as informaçóes oriundas de relatos dos indivíduos durante o período de maio a julho de 2013. Os entrevistados assinaram voluntariamente um Termo de Consentimento Livre e Esclarecido, declarando conhecimento sobre a utilização dos dados obtidos e ciência da garantia de omissão de dados de identificação.

O tamanho da amostra foi definido considerando-se erro de 0,05 , e erro amostral entre 4 e $0,8 \%$ para prevalências entre 50 e $1 \%$, respectivamente, com nível de confiança de 95\%. A prevalência de hipertensos e diabéticos em Governador Valadares foi estimada em Porto et al. ${ }^{1}$.

Inicialmente, as variáveis das entrevistas foram relativas à identificação e ao perfil sociodemográfico dos indivíduos, constando de informaçóes como idade, sexo, escolaridade, renda mensal, ocupaçáo e local de residência. Foram incluídos apenas aqueles que se autodeclararam diagnosticados com DM e/ou HAS e, em sequência, foi apurado quais os medicamentos anti-hipertensivos e antidiabéticos prescritos para o tratamento. Em relação ao estilo de vida, os indivíduos foram questionados quanto à prática de exercícios físicos, o tempo e a frequência de prática, e as modalidades.

O estudo foi executado sob autorização prévia da diretoria do Departamento de Atenção à Saúde/DAS da Secretaria Municipal de Saúde de Governador Valadares, tendo sido aprovado pelo Comitê de Ética de Pesquisa em Seres Humanos da Universidade Federal de Juiz de Fora (registro $\mathrm{n}^{\circ}$ 341.113), em conformidade com a Resoluçáo 466/2012 do Conselho Nacional de Saúde.

\section{Resultados e discussão}

O perfil sociodemográfico deste estudo, conforme será apresentado, corrobora os dados oficiais do DATASUS, que revelam maior acesso de mulheres e idosos no serviço público de saúde? ${ }^{9}$ Na Tabela 1 são apresentadas as características sociodemográficas dos 315 indivíduos entrevistados, em que a maioria era do sexo feminino $(78,6 \%)$, residentes em Governador Valadares ou em distritos (94\%), sendo uma minoria proveniente de outras cidades (6\%). Quanto à renda, $67,3 \%$ afirmaram receber um salário-mínimo. Este dado concorda com a ocupação autorreferida, uma vez que mais de um terço dos entrevistados eram aposentados (41\%) e no Brasil cerca de $71 \%$ deles recebem um salário-mínimo ${ }^{10}$. Nesse caso, é importante destacar que o nível socioeconômico mais baixo está associado a maior prevalência de HAS, com agravos em órgãos-alvo e eventos cardiovasculares ${ }^{2}$.
Observou-se também que menos de 30\% dos entrevistados tinham alguma ocupação, e 26\% eram "do lar". Compatível com a ocupação, a faixa etária dos entrevistados concentrou-se nos grupos de idades mais elevadas, de 51 anos a 80 anos, correspondendo a $82,2 \%$ da população entrevistada (Gráfico 1). Além disso, a maior parte dos usuários apresentava baixo nível de escolaridade, sendo $11,8 \%$ analfabetos e $38,4 \%$ com menos de quatro anos de estudo. Esses dados relativos à escolaridade muitas vezes podem representar um entrave para a eficácia de políticas públicas de saúde ou mesmo a adesão dos pacientes ao tratamento ${ }^{11}$.

Tabela 1: Características sociodemográficas dos indivíduos na amostra populacional estudada em Governador Valadares-MG, $2013(n=315)$.

\begin{tabular}{|c|c|c|}
\hline Variáveis sociodemográficas & $\mathbf{n}$ & $\%$ \\
\hline \multicolumn{3}{|l|}{ Sexo } \\
\hline Masculino & 73 & 23,1 \\
\hline Feminino & 242 & 76,9 \\
\hline \multicolumn{3}{|l|}{ Ocupação } \\
\hline Com ocupação & 91 & 28,9 \\
\hline Aposentado e/ou pensionista & 129 & 41,0 \\
\hline Do lar & 82 & 26,0 \\
\hline Sem ocupação & 5 & 1,6 \\
\hline Sem informação & 8 & 2,5 \\
\hline \multicolumn{3}{|l|}{ Renda mensal (SM) } \\
\hline$<1$ & 2 & 0,6 \\
\hline 1 & 212 & 67,3 \\
\hline 2 a 3 & 53 & 16,9 \\
\hline$\geq 4$ & 48 & 15,2 \\
\hline \multicolumn{3}{|l|}{ Escolaridade } \\
\hline Analfabeto & 37 & 11,8 \\
\hline$<4$ anos & 121 & 38,4 \\
\hline$\geq 4$ anos & 121 & 38,4 \\
\hline Sem informação & 36 & 11,4 \\
\hline \multicolumn{3}{|l|}{ Localizaçáo } \\
\hline Governador Valadares & 292 & 92,7 \\
\hline Distrito de G. Valadares & 4 & 1,3 \\
\hline Outra cidade de MG & 19 & 6,0 \\
\hline
\end{tabular}

SM: Salário-mínimo vigente à época da pesquisa $(1 \mathrm{SM}=\mathrm{R} \$ 678,00)$.

Do total de entrevistados, $47,6 \%$ se declararam diabéticos, e $83,8 \%$ hipertensos, sendo que $31,4 \%$ possuíam ambas as condiçóes. Entre os diabéticos e os hipertensos, $78,6 \%$ eram do sexo feminino, e $21,4 \%$ eram do sexo masculino, respectivamente. Nesse caso, há que se considerar o fato de que indivíduos do sexo feminino possuem maior tendência de apresentar HAS devido à mudança dos hábitos alimentares e da rotina, como o trabalho fora de casa, além da função do lar, mãe e esposa ${ }^{12}$. Enquanto isso, indivíduos do sexo masculino são resistentes em procurar o serviço médico ${ }^{13}$. 
Sendo assim, a baixa porcentagem de homens na amostra não representa necessariamente a incidência de DM e/ou HAS masculina no município. A alta prevalência de DM e HAS encontrada deve-se ao fato de que as entrevistas foram realizadas onde os pacientes buscam acompanhamento para essas doenças. Portanto, esses dados não representam a prevalência de diabéticos e hipertensos do município, mas de uma amostra in loco em Governador Valadares.

Como a etiologia da HAS e do DM é multifatorial, as estratégias de combate a essas doenças devem ser variadas com base em suas diversas configuraçóes. A necessidade primordial é proporcionar a detecção precoce para minimizar a incidência de futuras complicaçôes, tanto na populaçáo de alto risco, os idosos em que há elevada morbimortalidade pela $\mathrm{HAS}^{12}$, quanto na população em geral. Como a população entrevistada em Governador Valadares foi composta majoritariamente de idosos (61,9\%, Gráfico 1), sugere-se que o Programa Hiperdia Minas assista grupos de risco nesse município. Sendo assim, infere-se também uma abrangência pelo Hiperdia desses indivíduos mais vulneráveis às doenças crônicas abordadas. Tenta-se, portanto, conscientizá-los dessa realidade, além de buscar estratégias preventivas de educação em saúde e melhoria na qualidade de vida.

Gráfico 1: Distribuição percentual dos indivíduos de acordo com a faixa etária $(n=315)$. Os números absolutos são mostrados sobre as barras.

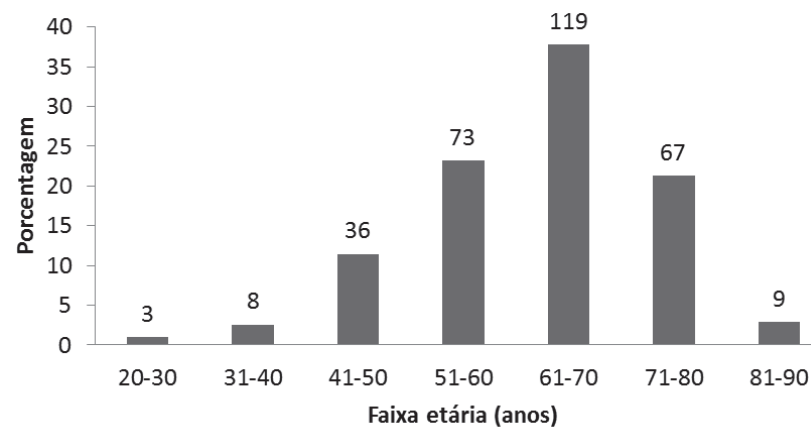

Existe uma relação direta e linear da pressão arterial com a idade, sendo a prevalência de HAS superior a $60 \%$ na faixa etária acima de 65 anos $^{2}$. Isso justifica a associaçáo entre indivíduos majoritariamente idosos e cuja doença crônica mais prevalente é a HAS à semelhança de outros inquéritos realizados em cidades brasileiras $^{12}$.

Uma alta taxa de usuários foi encontrada fazendo tratamento medicamentoso, sendo que todos os hipertensos faziam uso de pelo menos um anti-hipertensivo e $83,3 \%$ dos diabéticos usavam hipoglicemiantes. A respeito dos medicamentos para o tratamento de DM, a metformina constituía o hipoglicemiante mais prescrito pelos médicos, correspondendo a $48,4 \%$ das prescriçóes, o equivalente a $62 \%$ dos diabéticos medicados (Tabela 2). A glibenclamida e a insulina representaram $28,1 \%$ e $19,3 \%$ das prescriçóes, respectivamente; sendo que 54\% dos diabéticos usavam a glibenclamida e $24,7 \%$ fazia uso da insulina. O uso concomitante de insulina e outros antidiabéticos sugere uma maioria de pacientes apresentando diabetes do tipo 2 com glicemia não controlada. $\mathrm{Na}$ Tabela 2 também são apresentados os anti-hipertensivos utilizados pelos entrevistados. O losartano foi o fármaco mais usado pela amostra populacional diabética e/ou hipertensa, correspondendo a $27,8 \%$ das prescriçóes e a $39,4 \%$ dos hipertensos medicados, seguida da hidroclorotiazida $(23,9 \%)$, captopril $(22 \%)$ e propranolol $(13,3 \%)$. Uma menor porcentagem de hipertensos fazia o uso de bloqueadores de canais de cálcio $(12,2 \%)$ ou de outros anti-hipertensivos (4,9\%), que compreendem a metildopa, clonidina, alisquireno e hidralazina etc.

Tabela 2: Antidiabéticos utilizados pelos diabéticos ( $n=150)$ e anti-hipertensivos utilizados pelos hipertensos $(n=264)$ da amostra populacional estudada em Governador Valadares-MG, 2013.

\begin{tabular}{|c|c|c|c|}
\hline Antidiabético & $\begin{array}{c}n \text { de } \\
\text { prescriçóes }\end{array}$ & $\begin{array}{c}\% \text { das } \\
\text { prescriçóes }\end{array}$ & $\begin{array}{l}\% \text { entre os } \\
\text { diabéticos }\end{array}$ \\
\hline Metformina & 93 & 48,4 & 62 \\
\hline Glibenclamida & 54 & 28,1 & 54 \\
\hline Insulina & 37 & 19,3 & 24,7 \\
\hline Outros & 8 & 4,2 & 5,3 \\
\hline TOTAL & 192 & $\begin{array}{c}100 \% \\
(\mathrm{n}=192)\end{array}$ & $100 \%(n=150)$ \\
\hline Anti-hipertensivo & $\begin{array}{c}n \text { de } \\
\text { prescriçóes }\end{array}$ & $\begin{array}{c}\% \text { das } \\
\text { prescriçóes }\end{array}$ & $\begin{array}{c}\% \text { entre os } \\
\text { hipertensos }\end{array}$ \\
\hline \multicolumn{4}{|l|}{ iECA } \\
\hline Captopril & 58 & 15,5 & 22,0 \\
\hline Enalapril & 16 & 4,3 & 6,1 \\
\hline Lisinopril & 1 & 0,3 & 0,4 \\
\hline \multicolumn{4}{|l|}{ BRA } \\
\hline Losartano & 104 & 27,8 & 39,4 \\
\hline Valsartano & 3 & 0,8 & 1,1 \\
\hline Olmersatano & 2 & 0,5 & 0,8 \\
\hline \multicolumn{4}{|l|}{ Diuréticos } \\
\hline Hidroclorotiazida & 63 & 16,9 & 23,9 \\
\hline Furosemida & 16 & 4,3 & 6,1 \\
\hline Espironolactona & 9 & 2,4 & 3,4 \\
\hline \multicolumn{4}{|l|}{$\beta$-bloqueadores } \\
\hline Propranolol & 35 & 9,4 & 13,3 \\
\hline Atenolol & 16 & 4,3 & 6,1 \\
\hline Metoprolol & 2 & 0,5 & 0,8 \\
\hline Carvedilol & 4 & 1,1 & 1,5 \\
\hline \multicolumn{4}{|l|}{ BCC } \\
\hline Nifedipino & 20 & 5,4 & 7,6 \\
\hline Anlodipino & 10 & 2,7 & 3,8 \\
\hline Verapamil & 2 & 0,5 & 0,8 \\
\hline Outros & 13 & 3,5 & 4,9 \\
\hline TOTAL & 374 & $\begin{array}{c}100 \% \\
(n=374)\end{array}$ & $100 \%(n=254)$ \\
\hline
\end{tabular}

iECA: inibidor da enzima conversora de angiotensina; BRA: bloqueadores do receptor $\mathrm{AT}_{1}$ de angiotensina-II; BCC: bloqueadores de canais de cálcio. 
É importante destacar que o total de medicamentos anti-hipertensivos e antidiabéticos prescritos aos entrevistados é superior ao número de indivíduos medicados, porque havia associaçóes de fármacos em um mesmo medicamento ou prescriçóes de múltiplos fármacos, caracterizando polifarmácia. A abordagem farmacológica de doenças crônicas, como a HAS e o DM, exige atenção especial dos prescritores, uma vez que o uso concomitante de medicamentos antidiabéticos pode atuar de forma sinérgica sobre a redução dos níveis glicêmicos, aumentando a incidência de episódios de hipoglicemia, os quais são potencialmente fatais ${ }^{3}$. Quanto à combinação de anti-hipertensivos, deve-se evitar a combinação de fármacos com mecanismos de ação similares, exceto na associação de diuréticos tiazídicos e de alça com poupadores de potássio ${ }^{14}$. Ainda, 31,4\% dos indivíduos eram hipertensos e diabéticos, e, possivelmente faziam uso concomitante de anti-hipertensivos e antidiabéticos. A associação desses fármacos pode gerar possíveis interaçóes medicamentosas, potencializando ou reduzindo o efeito hipoglicemiante. $\mathrm{O}$ uso de antidiabéticos com inibidores da enzima conversora de angiotensina (iECA), como o captopril, por exemplo, pode potencializar o efeito hipoglicemiante; enquanto o uso do diurético hidroclorotiazida com medicamentos antidiabéticos pode aumentar a glicemia, diminuindo o efeito hipoglicemiante ${ }^{15}$.

A maioria dos medicamentos antidiabéticos e anti-hipertensivos citados pelos entrevistados encontram-se na Relação Nacional de Medicamentos Essenciais (Rename), exceto o lisinopril, valsartano e olmesartano ${ }^{16}$. Entre as estratégias para o uso racional de medicamentos, a elaboração e utilização de uma lista de medicamentos essencial favorece a qualidade da atenção à saúde, melhora a gestão de medicamentos, facilita o treinamento do prescritor e melhora a informação ao paciente, além de ser um instrumento para a gestáo pública que padroniza condutas e possibilita economia ${ }^{17}$. Encontrar uma porcentagem, mesmo que pequena, de prescriçóes de medicamentos não essenciais em uma população de renda eminentemente baixa foi surpreendente, uma vez que pode comprometer a adesão ao tratamento.

A rede de atenção primária à saúde, que no Brasil se dá, principalmente, através da ESF, é vista como um sistema que busca a prevenção dessas condiçôes clínicas, proporcionando a detecção precoce e o tratamento eficaz. O Programa Hiperdia possibilitou o cadastramento e acompanhamento dos indivíduos diabéticos e/ ou hipertensos captados e vinculados às UBS ou ESF do SUS. De acordo com os dados do SisHiperdia ${ }^{7}$, Governador Valadares apresenta 6,53\% diabéticos cadastrados, sendo 29,17\% cadastrados como diabéticos com hipertensão. O Hiperdia funciona nas UBS e também na Policlínica Municipal de Governador Valadares, local de realizaçáo das entrevistas e onde os pacientes fazem acompanhamentos por meio de consultas com os médicos especialistas. Além disso, a atenção primária atua garantindo acesso aos medicamentos por meio das farmácias presentes nos postos de saúde e acompanhamento pelos agentes de saúde e médicos, favorecendo um tratamento eficaz, o que reduz os riscos de possíveis complicaçóes. Estima-se que o custo anual com medicamentos para a HAS corresponda a 52,3\% dos custos para o tratamento dessa doença no SUS ${ }^{18}$. Esse ônus, se não fosse subsidiado pelo Governo, inviabilizaria a contemplação de grande parcela da população brasileira, já que a gratuidade de medicamentos assegura maior adesão ao tratamento.

Gráfico 2: Distribuição dos indivíduos quanto à prática de atividade física $(A, n=315)$, frequência semanal $(B, n=132)$ e tempo de atividade física $(\mathrm{C}, \mathrm{n}=132)$.
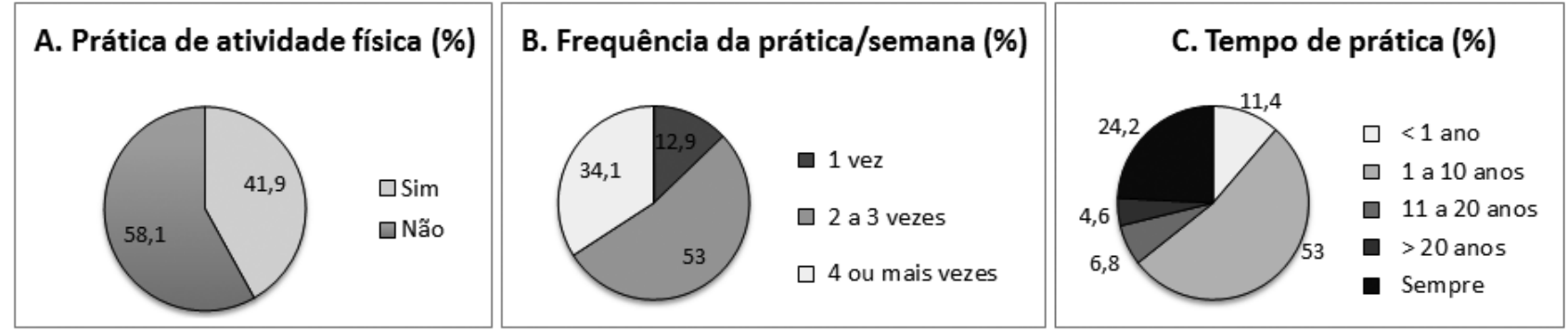

O Gráfico 2 e a Tabela 3 mostram as variáveis quanto à prática de exercícios físicos. Um pouco menos da metade dos usuários entrevistados $(41,9 \%)$ declarou praticar alguma atividade física (Gráfico $2 \mathrm{~A}$ ), sendo a maioria dos praticantes pertencente ao sexo feminino $(75,7 \%)$. Os participantes que se declararam praticantes, faziam atividade física com alta regularidade (Gráfico 2B), incluindo frequência semanal de 2 a 3 vezes (53\%) ou superior a 4 vezes $(34,1 \%)$. Além disso, observou-se que a preocupação com a atividade física não era recente, pois a maioria relatou fazê-la de 1 a 10 ou mais anos (53\%) e poucos praticavam há menos de 1 ano (11,4\%, Gráfico 2C). 
Tabela 3: Modalidades esportivas praticadas pelos indivíduos na amostra populacional estudada em Governador ValadaresMG, $2013(n=132)$.

\begin{tabular}{|c|c|c|}
\hline Modalidade & $\mathbf{n}$ & $\%$ \\
\hline Alongamento e/ou ginástica localizada & 15 & 11,3 \\
\hline Boliche & 3 & 2,2 \\
\hline Caminhada & 87 & 65,9 \\
\hline Ciclismo & 13 & 9,8 \\
\hline Dança & 10 & 7,6 \\
\hline Futebol & 1 & 0,7 \\
\hline Hidroginástica e/ou natação & 29 & 22 \\
\hline Basquete e/ou lancebol & 2 & 1,5 \\
\hline Musculação & 2 & 1,5 \\
\hline Pilates & 1 & 0,7 \\
\hline Voleibol & 1 & 0,7 \\
\hline
\end{tabular}

Conforme mostrado na Tabela 3, a modalidade de atividade física com maior adesão foi a caminhada $(65,9 \%)$, seguida da hidroginástica e/ou natação $(22 \%)$ e das atividades de alongamento e ginástica localizada $(11,3 \%)$. As menos citadas, em ordem decrescente, foram ciclismo, dança, boliche, basquete, musculação, futebol, pilates e voleibol. Vale ressaltar que alguns entrevistados relataram a prática de duas ou mais modalidades, contabilizando 164 citaçóes. Nesse sentido, de forma geral, é importante observar que a caminhada, como modalidade mais citada, apresenta baixo custo em relação às demais. Essa realidade pode estar associada com a baixa renda mensal dos entrevistados.

Há evidências de que alteraçôes no estilo de vida, com ênfase na alimentação e na prática de atividade física, estão associadas à prevalência de DM. Dados do Programa de Prevenção de Diabetes mostraram redução de $58 \%$ da incidência de DM mediante estímulo à dieta saudável e prática de atividade física ${ }^{19}$. Em relação à HAS, estudos clínicos revelam que os efeitos anti-hipertensores das modificaçóes do estilo de vida podem ser equivalentes à monoterapia com medicamentos ${ }^{20}$. Existem consensos na literatura e evidências clínicas de que o exercício físico aeróbio, de baixa e moderada intensidades, como a caminhada, se praticado de 3 a 5 vezes semanalmente por cerca de 30 minutos, reduz os níveis de pressão arterial dos pacientes hipertensos ${ }^{21}$. Além disso, no caso de idosos, também colabora para a melhoria da aptidão física, diminuição da gordura corporal, melhora da estética e ampliação da sociabilidade ${ }^{22}$.

Embora não seja possível determinar uma associação direta entre a atividade física e a redução dos fatores de risco para a HAS e o DM na populaçáo estudada, observou-se que essa medida não medicamentosa é adotada apenas pela metade dos usuários do Hiperdia. Também faz parte das estratégias do Hiperdia promover hábitos de vida saudáveis, como controle do tabagismo e a prática de atividades físicas, com o objetivo de diminuir alguns dos fatores de risco para a HAS e o DM. Nesse sentido, é importante direcionar estratégias para aumentar a parcela da população fisicamente ativa em Governador Valadares, pois há modalidades economicamente viáveis e comprovadamente eficazes, como discutido. Entretanto, essa prática só é possível mediante a formação de uma equipe de saúde multidisciplinar, que conte com profissionais que atuem em todos os âmbitos da saúde. No caso desse município, fica clara a necessidade de um serviço de atenção farmacêutica, educação física e nutrição. Para tanto, não só as gerências municipais, mas a política da ESF deve considerar a inclusão definitiva desses profissionais (farmacêuticos, educadores físicos, fisioterapeutas e nutricionistas) nas equipes básicas que assistam o Hiperdia local.

\section{Conclusão}

Diante dos dados coletados, é possível caracterizar o usuário do Programa Hiperdia Minas em Governador Valadares, como um indivíduo suscetível, pois é idoso, polimedicado, com baixo grau de instrução e de poder aquisitivo. Nesse sentido, o Hiperdia cumpre seu papel, pois o usuário atingido realmente carece desse serviço. Por outro lado, há que se chamar atenção para o fato de que, aparentemente, a intervenção terapêutica da equipe de saúde é eminentemente baseada em medicação, haja vista que quase todos os doentes eram tratados. Contudo, ao serem questionados sobre a prática de atividade física, averiguou-se que metade dos indivíduos era sedentária. Assim, estratégias que visem ao incentivo e orientação à atividade física devem ser implementadas pela equipe de saúde do município, estimulando os acometidos a aderirem ao tratamento e às mudanças no estilo de vida. Esse tipo de meta só é atingido com uma equipe de saúde multidisciplinar que envolva profissionais da saúde com diferentes formações. Um estudo pelo nosso grupo de pesquisa e extensão mostrou que a atuação conjunta de diferentes profissionais de saúde e sociólogos tem um impacto positivo sobre a qualidade de vida de idosos ${ }^{23}$.

Por fim, é importante destacar que os dados deste estudo foram obtidos por meio de informaçôes primárias, o que pode constituir uma limitação, uma vez que foram contabilizados como hipertensos e/ou diabéticos aqueles que assim se declaravam, sem testes clínicos para comprovar o diagnóstico dessas doenças. Além disso, as informações sociodemográficas e a respeito dos medicamentos utilizados ou estilo de vida não foram verificadas. É aceito, porém, que inquéritos populacionais baseados em informaçóes primárias permitam um diagnóstico rápido dos serviços de saúde, além de levar 
em conta a percepção do usuário sobre sua condição de saúde ${ }^{24,25}$. Ademais, utilizando informaçóes secundárias do serviço de atenção primária à saúde sobre a populaçáo hipertensa e diabética em Governador Valadares ${ }^{1}$, um estudo anterior mostrou resultados semelhantes aos encontrados neste trabalho, corroborando para a validação dos dados aqui descritos.

\section{Agradecimentos}

Aos estudantes envolvidos na coleta e tabulação dos dados (Barbara A. S. Reis, Douglas V. O. Santos, Jéssica M. S. Nery, Joandey C. Rocha, Layrane F. Albertino, Leocádia C. V. Zuba, Lília D. Castro, Lucas S. Boaventura, Luísa L. R. Oliveira, Maiara R. Salvador, Nadson Z. Leão, Natara H. A. Santos e Pedro H. M. Rezende), especialmente a Beatriz S. S. Araújo, Felipe C. Cavalcante, Fernanda M. Ribeiro, José A. C. Corrêa, Louise L. Santos, Marcos Vinícius F. Souza e Olívia M. R. P. Alves. À Prefeitura Municipal de Governador Valadares / Secretaria Municipal de Saúde (Ana M. Germano, Fernanda M. D. Rocha e Késia S. Pereira).

\section{Referências}

1. Porto LK, Cadete LV, Nascimento MBPG, Freire MN, Dias WT, Almeida NAV. Perfil epidemiológico de idosos e hipertensos e/ou diabéticos de Unidades da Estratégia de Saúde da Família/ESF, do município de Governador ValadaresMG. Rev Cient FACS 2011;13:87-92.

2. VI Diretrizes Brasileiras de Hipertensão. Arq Bras Cardiol. 2010;95(1):I-III.

3. Sociedade Brasileira de Diabetes. Diretrizes da Sociedade Brasileira de Diabetes: 2013-2014. São Paulo: AC Farmacêutica; 2014.

4. DATASUS. Informação de Saúde, Indicadores e Dados Básicos - Brasil - 2012, Indicadores de fatores de risco e de proteção. Brasília; 2012.

5. Malfatti CRM, Assunção AN. Hipertensão arterial e diabetes na Estratégia de Saúde da Família: uma análise da frequência de acompanhamento pelas equipes de Saúde da Família. Ciênc Saúde Coletiva. 2011;16(Supl.1):1383-8.

6. Muller M, Hermes GB, Flores LM. Perfil epidemiológico dos pacientes cadastrados no Sistema Hiperdia do município de Santa Maria, RS. In: 27a Salão de Iniciação Científica da JAI - Jornada Acadêmica Integrada; 2012; Santa Maria. Santa Maria: UFSM; 2012. p. 1-5.

7. SisHiperdia, Sistema de gestão clínica de hipertensão arterial e diabetes mellitus da atenção básica. Brasília; 2013.

8. Instituto Brasileiro de Geografia e Estatística. Censo Demográfico. Brasília; 2010.

9. Carvalho ALM, Leopoldino RWD, Silva JEG, Cunha CP.
Adesão ao tratamento medicamentoso em usuários cadastrados no Programa Hiperdia no município de Teresina (PI). Ciênc Saúde Coletiva. 2012;17(7):1885-92.

10. ANASPS confirma que entre 2005 e 2013 mais de cinco milhóes de benefícios caíram pra faixa do salário mínimo. Imprensa da ANASPS, Brasília, 20 fev. 2014 [acesso em 8 ago.]. Disponível em: http://www.anasps.org.br/mostra_ materia.php?id=3748

11. Gusmão JL, Ginani JF, Silva GV, Ortega KC, Mion JrD. Adesão ao tratamento em hipertensão arterial sistólica isolada. Rev Bras Hipertens. 2009;16(1):38-43.

12. Piati J, Felicetti CR, Lopes AC. Perfil nutricional de hipertensos acompanhados pelo Hiperdia em Unidade Básica de Saúde de cidade paranaense. Rev Bras Hipertens. 2009;16(2):123-9.

13. Figueiredo W. Assistência à saúde dos homens: um desafio para os serviços de atenção primária. Ciênc Saúde Coletiva. 2005;10(1):105-9.

14. Duarte OO, Faria WRC, Pinto FM, Nakaoka VYES, Kashiwabara TGB. Tratamento ambulatorial da hipertensão arterial sistêmica - revisão de literatura. Rev UNINGÁ. 2014;17(2):22-9.

15. Amaral DMD, Perassolo MS. Possíveis interações medicamentosas entre os anti-hipertensivos e antidiabéticos em participante do Grupo HIPERDIA de Paropé, RS (Uma análise teórica). Rev Ciênc Farm Básica Apl. 2012;33(1):99-105.

16. Brasil. Ministério da Saúde. Secretaria de Ciência, Tecnologia e Insumos Estratégicos. Departamento de Assistência Farmacêutica e Insumos Estratégicos. Relação Nacional de Medicamentos Essenciais: Rename 2013. Brasília; 2013.

17. Brasil. Ministério da Saúde. Secretaria de Ciência, Tecnologia e Insumos Estratégicos. Uso racional de medicamentos: temas selecionados. Brasília; 2012.

18. Marinho MGS, Cesse EAP, Bezerra AFB, Sousa IMC, Fontbonne A, Carvalho EF. Análise de custos da assistência à saúde aos portadores de diabetes melito e hipertensão arterial em uma unidade de saúde pública de referência em Recife - Brasil. Arq Bras Endocrinol Metab. 2011;55(6):406-11.

19. Barceló A, Aedo C, Rajpathak S, Robles S. The cost of diabetes in Latin America and the Caribean. Bull World Health Organ. 2003;81(1):19-27.

20. Elmer PJ, Obarzanek E, Vollmer WM, Simons-Morton D, Stevens VJ, Young DR, Lin PH, Champagne C, Harsha DW, Svetkey LP, Ard J, Brantley PJ, Proschan MA, Erlinger TP, Appel LJ. Effects of comprehensive lifestyle modification on diet, weight, physical fitness and blood pressure control: 18-month results of a randomized trial. Ann Intern Med. 2006;144(7):485-95.

21. Laterza MC, Amaro G, Negrão CE, Rondon MUPB. Exercício físico regular e controle autonômico na hipertensão arterial. Rev SOCERJ. 2008;21(5):320-8.

22. Krug RR, Marchesan M, Conceição JCR, Mazo GZ, 
Antunes GA, Romitti JC. Contribuiçôes da caminhada como atividade física de lazer para idosos. Licere. 2011;14(4):1-29.

23. Faria LR, Calabria LK, Silva CLA, Barbosa MCA, Santo RPE, Cau SBA. Atenção preventiva e educativa em saúde do idoso: uma proposta de integração dos saberes e práticas. Estud Interdiscipl Envelhec. 2015;20:9-32.
24. Chrestani MAD, Santos IS, Matijasevich AM. Hipertensão arterial sistêmica autorreferida: validação diagnóstica em estudo de base populacional. Cad Saúde Pública. 2009;25(11):2395-406.

25. Jardim R, Barreto SM, Giatti L. Autorrelato e relato de informante secundário na avaliação da saúde em idosos. Rev Saúde Pública. 2010;44(6):1120-9.

\section{Como citar este artigo:}

Saraiva LGF, Dornelas PG, Cau SBA, Calábria LK. Perfil epidemiológico de pacientes atendidos em uma rede ambulatorial do Hiperdia Minas em Governador Valadares-MG. Rev. Aten. Saúde. 2016;14(48):40-47. 